\title{
Superando el síndrome del "enemigo externo" en una firma autopartista a través del kaizen
}

\author{
Jorge lván Pérez Rave ${ }^{\mathrm{a} *}$, Daniel Andrés La Rotta Forero ${ }^{\mathrm{b}}$, Carlos Andrés Vera Garcés ${ }^{\mathrm{c}}$ \\ a*ejipr056@udea.edu.co, UdeA, Colombia \\ bdlarotta@udea.edu.co, UdeA, Colombia \\ ccarlosvera_g@hotmail.com, UdeA, Colombia
}

\begin{abstract}
Resumen
Dos problemas de una firma autopartista, con causas atribuidas inicialmente a factores externos no controlables (síndrome del "enemigo externo"), motivan el uso del kaizen, incorporando concepciones filosóficas y metodológicas. La metodología propuesta consta de tres etapas; la primera, iniciar, pretende conducir hacia el compromiso, la participación y el desarrollo de habilidades iniciales de los empleados; luego sigue desarrollar, que ejecuta el análisis y la solución del problema; se culmina con sostener, para mantener las contramedidas, evitar la recurrencia del problema e incentivar a los participantes. Al desplegar la metodología en los dos casos objeto de estudio (1. Muda de espera por falta de suministro y 2. Muda de defectos de hojas impresas), se identificó que las causas de dichos muda se debieron a factores internos a la empresa y no a las creencias iniciales de la firma sobre aspectos externos, no controlables. La causa del muda de espera estuvo relacionada con la cantidad de personal programado en la línea de ensamble; las impresiones defectuosas se asociaron a la política de almacenaje y protección de las hojas, así como a las instrucciones de impresión. El impacto del despliegue en la empresa se dedujo satisfactorio, ya que mediante la implantación de contramedidas basadas en creatividad, sentido común, consenso y participación de los empleados, se favorecen indicadores asociados a los problemas precisos, entre ellos, costos anuales de los muda (reducción: $87 \%$ en costos del muda de espera y 60,5\% en costos de hojas defectuosas). Este trabajo provee elementos teóricos y metodológicos, que posibilitan la adaptación a otros contextos, exigiendo: compromiso directivo, justificación del cambio, entrenamiento de empleados, incentivos, comunicación y estrategias de aprendizaje, entre otros, para solucionar problemas y sostener los eventos kaizen.
\end{abstract}

Palabras clave

Kaizen. Investigación aplicada. Mejoramiento continuo. Mudas de manufactura.

\section{Introducción}

Nadie puede manotear bien la pelota en ese campo, o "siempre hallarán un factor externo a quien culpar", son frases que cita Peter Senge cuando expone al "enemigo externo" como una de las siete barreras de aprendizaje (SENGE, 2005). Este sindrome, de posible aparición ante problemas empresariales, refleja un pensamiento asistémico, que interrumpe toda intención de superar las oportunidades de mejora, debido a la creencia de imposibilidad de control interno.

Una firma autopartista Colombiana, denotada por las letras $\mathrm{ABC}$, muestra síntomas de esta barrera de aprendizaje, al verse "obligada" a sobrevivir con dos mudas (término Japonés que representa los desperdicios de manufactura). Una de ellas es muda de espera por falta de suministro, de manera recurrente y por tiempo prolongado, en una de sus líneas de producción. El asocio de este problema con el síndrome del "enemigo externo", radica en la aceptación, por parte de la empresa, de que la causa del mismo es por "culpa de la empresa cliente (ensambladora)", la cual, por estrategia consensuada de reducción de costos y tiempos, se responsabiliza de importar el suministro que necesita su proveedor $\mathrm{ABC}$ para despachárselo paulatinamente. El síndrome del "enemigo externo", en palabras de la empresa, puede representarse por medio de la siguiente frase: 
“a pesar de que el cliente tiene el suministro en su planta, no nos envía el material a tiempo, y por ello, nos toca esperar". El otro muda corresponde a defectos en algunas de las hojas que se imprimen en la empresa y cuya causa, a priori, es asociada al "enemigo externo": "la impresora vino mala".

Con base en lo anterior y en busca de evidencias soportadas en datos y hechos, el problema objeto de estudio fue sistematizado por los autores, a través de las siguientes preguntas de investigación: ¿cuáles son las causas del muda de espera por falta de suministro y del muda defectos de hojas impresas, en el ámbito de la empresa $A B C$ ?, ¿estas causas se deben a factores externos o internos a la empresa?, de ser internos ¿cómo intervenir dichas causas, a fin de eliminar o reducir la recurrencia?

La principal perspectiva teórica, no exclusiva, adoptada para buscar solución al problema de investigación expuesto, corresponde al kaizen, puesto que, como se podrá ver en el marco conceptual y en la revisión literaria, existe evidencia sobre la eficacia de esta filosofía para llegar a la causa raíz de problemas manufactureros, pero a diferencia de otras herramientas, como por ejemplo, el análisis de falla, el kaizen se enfoca en la implementación de acciones tipo "paso a paso", basadas en su mayoría en el sentido común, brindando participación activa del nivel operativo, lo cual es una directriz de la empresa donde se hizo el estudio.

En este trabajo, además, se pretende vincular la experiencia y las opiniones de los trabajadores, con una componente objetiva basada en datos y hechos, para dar solución al problema en tiempo relativamente corto, propiciando el aprendizaje del nivel operativo y facilitando la reproducibilidad de las etapas seguidas.

En el segundo apartado se presenta un referencial teórico del kaizen, que incluye su conceptualización, así como una revisión literaria que describe los 10 estudios más citados entre 2006-2010, bajo un algoritmo específico de búsqueda en Scopus. El tercero presenta la metodología propuesta para responder a las preguntas de investigación, el cuarto ofrece los resultados del despliegue en una firma autopartista, el quinto se dedica a la discusión de los mismos, el sexto presenta las conclusiones, luego vienen los agradecimientos y se finaliza con las referencias bibliográficas.

\section{Referencial teórico}

\subsection{Conceptualización del Kaizen}

El término kaizen, acuñado en la década de los ochenta por Masaaki Imai, hace parte de la competitividad del sistema de producción japonés (SUÁREZ; MIGUEL, 2008). Diversos autores como
Brunet, y New (2003), afirman que, a pesar de su importancia y de los diversos esfuerzos por definirlo (IMAl, 1986; WITTENBERG, 1994; BRUNET; NEW, 2003), existe en la literatura cierta ambigüedad en su conceptualización. En términos generales, puede afirmarse que el kaizen ha sido interpretado como una herramienta de mejoramiento empresarial, pero también como filosofía de negocio e incluso de vida (IMAl, 1986; WITTENBERG, 1994). Los esfuerzos tipo kaizen requieren que los miembros de la empresa se comprometan con el mejoramiento gradual y constante, con el fomento de la participación de los empleados (BESSANT, 2003; WOMACK, JONES; ROOS, 1990) y con valores como la disciplina, el orden y el rechazo al desperdicio. Se enfatiza en la importancia del apoyo y compromiso de la alta dirección para brindar los recursos y para fomentar la participación y la autonomía de los equipos de trabajo. En general, el kaizen hace de la simplicidad su mayor fortaleza, apoyándose especialmente en el sentido común, en la motivación y en el empeño de los empleados. Por otro lado, visto como potencialidad, hace entender que las posibilidades de mejora dentro de una empresa son eternas e infinitas (OHNO, 1978). El kaizen contribuye a que la organización se enfoque en la reducción del muda (desperdicio). Para lograrlo se apoya en técnicas como: ciclo $\mathrm{P}-\mathrm{H}-\mathrm{V}-\mathrm{A}$, siete herramientas básicas de calidad, $5 \mathrm{~S}$ y estandarización de procesos, todo ello, fundamentado en la capacitación de los miembros de la organización. Históricamente se han realizado esfuerzos por llevar los exitosos resultados del kaizen a empresas fuera de Japón (AOKl, 2008; BARRAZA; SMITH; DAHLGARD, 2009; AL-TAHAT; ETEIR, 2010) encontrando que la cultura en la que se movilizarán las nuevas prácticas se convierte en un factor potenciador o inhibidor de los resultados (LANDA, 2009).

La filosofía kaizen ha potenciado con el tiempo la aparición de los "eventos kaizen", llamados también "kaizen blitz" o "Workshops"; es decir, proyectos enfocados y estructurados, que a través de la dedicación de un equipo multidisciplinario y en un marco de tiempo acelerado, contribuyen a mejorar un área objeto de estudio (FARRIS et al., 2008). Los eventos kaizen buscan entrenar a los participantes en las herramientas de mejora, implementar cambios y generar una cultura propicia para el mejoramiento continuo (IMAl, 1997). Exigen una etapa previa de preparación y una posterior en la que se hace seguimiento a los resultados, todo ello articulado a través de un facilitador. Al abordar la solución de problemas, el eje central del proceso de reflexión es el ciclo P-H-V-A, enmarcado en el método de análisis y solución de problemas (MASP); en la etapa de planeación se define y analiza el problema, se estudian sus causas y se establecen las contramedidas (¿el qué, el por qué y el cómo?), en la etapa del 
hacer se implementan las contramedidas, en la de verificar se confirma el resultado, y en la de actuar se estandarizan los procesos (IMAl, 2001; AGUDELO; ESCOBAR, 2007). A pesar de la importancia de estos eventos, existen inquietudes con respecto a aquellos elementos que los hacen exitosos (FARRIS et al., 2008; LANDA, 2009). Aspectos como la cultura organizacional, la calidad del facilitador (fomento del interés por participar), la configuración del equipo, los procesos de comunicación y la justificación del cambio, parecen potenciar los resultados alcanzados y su sostenimiento (LANDA, 2009). Por otro lado, la ausencia de claridad en el método de comunicación y la justificación de los objetivos, la ausencia de representatividad de alguna función clave y la falta de autonomía del equipo, tienden a explicar algunos de sus fracasos (FARRIS et al., 2008).

\subsection{Top 10 de estudios más citados en Scopus sobre Kaizen (2006-2010)}

En esta sección se describen los trabajos que forman parte del Top 10 de los estudios, que tratan el kaizen como eje central, más citados por la comunidad científica entre 2006-y 2010. La consulta fue realizada en Scopus ${ }^{\circledR}$, uno de los buscadores científicos más posicionados (CODINA, 2005), empleando el siguiente algoritmo: término: "kaizen"; lugar: título; tipo de documentos: artículos y paper de conferencia; periodo: 2006-2010; áreas: ingenierías y, negociosadministración-contabilidad; fecha de consulta: 02-04-2010; total de documentos en el top: 10; total de documentos arrojados por el algoritmo: 41.

Alukal (2007) resume las principales técnicas del kaizen que han contribuido al éxito de Toyota. Plantea algunas de sus particularidades, entre ellas, fomentar una cultura en la que el recurso primordial sea la inteligencia, la creatividad y la generación de ideas de sus empleados, en lugar de simplemente sus manos. Se reitera la necesidad de extender la filosofía a sus proveedores, construyendo relaciones de largo plazo. Afirma que el objetivo principal del Sistema de Producción Toyota no es la manufactura esbelta o el kaizen, sino el poder alcanzar los objetivos organizacionales, beneficiando a la sociedad, equilibrando las metas a corto y largo plazo y alineando los intereses de las persona con los de la compañía. Aoki (2008) estudia la incidencia de las prácticas administrativas al transferir actividades kaizen a nueve plantas autopartistas japonesas en China. Concluye que en los casos exitosos, tienden a destacarse prácticas que favorecen los sistemas de sugerencias provenientes de equipos, la generación de trabajadores capaces de desempeñar más de un rol, los empleos a largo plazo y la presencia diaria del administrador en el "piso" para verificar la evolución del trabajo. Resalta la necesidad de facilitar la comunicación inter-funcional, de desarrollar la iniciativa del trabajador, así como su disciplina.

En Farris et al. (2008), identifican las características que determinan los fracasos de los eventos kaizen, en términos de sus resultados iniciales y de su sostenimiento en el tiempo. Afirman que existe debilidad en la literatura, pues no se han desarrollado suficientes estudios que den cuenta de ello. Concluyen que la ausencia de claridad en el método de comunicación y la justificación de los objetivos, la ausencia de representatividad de alguna función clave y la falta de autonomía del equipo, tienden a explicar algunos de los fracasos estudiados. Doolen et al. (2008) describen la aplicación de una metodología para medir y evaluar el impacto de los eventos kaizen en el desempeño de la organización, incluyendo los resultados en el talento humano. Estudian dos eventos kaizen dentro de una misma organización. Encuentran que el apoyo administrativo es fundamental para obtener resultados en las actitudes del recurso humano, destacando que estos logros no se traducen automáticamente en mejoras sostenidas.

Farris et al. (2009), afirman que existen pocos estudios que permiten concluir cuáles son aquellos insumos y procesos que determinan la bondad de los resultados de los eventos kaizen. Por ello, estudian 51 experiencias en seis organizaciones manufactureras. Identifican aquellos factores que inciden en el desarrollo de capacidades actitudinales y de resolución de problemas entre el personal. Barraza, Smith y Dahlgard (2009) afirman que la literatura académica viene desarrollando una especial curiosidad por estudiar las experiencias de aplicación del pensamiento esbelto (lean thinking) o del kaizen en el sector público. El objetivo del artículo es mostrar la manera como estas metodologías están siendo abordadas en España. Concluyen que las técnicas más usadas son las $5 \mathrm{~S}$, los eventos kaizen y el mapeo de procesos.

Marin, Garcia y Bonavia (2009) exploran la posibilidad de mejorar los indicadores de producción desarrollando eventos kaizen. A través de equipos en los que participan administradores y personal operativo se busca la implementación de mejoras en 11 empresas automovilísticas. En cada compañía se desarrollan diversas intervenciones durante 9 a 12 meses. Los autores presentan la situación inicial, las actividades realizadas y la evolución del desempeño de las mismas. Murata y Katayama (2010) presentan un procedimiento para la construcción de casos de estudio kaizen, basado en experiencias anteriores. Este consta esencialmente de dos pasos; en el primero se construye una "base de datos" de casos kaizen. Allí se identifican diversos ejercicios, se analizan profundizando en sus motivaciones y aspectos indagados, así como en sus relaciones. En 
el segundo paso, se pone en uso la base de datos construida, para desarrollar el nuevo caso.

Al-Tahat y Eteir (2010) analizan la habilidad de las compañías manufacturera de Jordania para aplicar los principios del mejoramiento continuo. Para lograrlo estudian dos empresas del sector industrial, que han implementado efectivamente el kaizen, identificando las ventajas y los cambios logrados. lgualmente, indagan en 105 compañias manufactureras la posibilidad futura de implementar dicha filosofía. Suárez y Ramis (2010) presentan un ejemplo exitoso de la manera como el lean-kaizen es implementado para contribuir al mejoramiento del servicio brindado por el talento humano en una organización pública mexicana, identificando elementos potenciadores e inhibidores.

\section{Metodología}

La Figura 1 ofrece la metodología propuesta para responder a las preguntas de investigación, tomando como base lecciones aprendidas de los autores y de los diferentes estudios de referencia. Esta consta de tres etapas: iniciar, desarrollar y sostener, las cuales forman un ciclo con posibilidad de ejecutarse de manera interminable, siendo ello deseable, de acuerdo con los postulados filosóficos del concepto kaizen.

\section{Despliegue de la metodología}

A continuación, los resultados se presentan según los momentos metodológicos expuestos en la Figura 1, resaltando que el rol de facilitador estuvo a cargo del equipo investigador, bajo el apoyo y compromiso de la dirección de planta de la empresa y los jefes de los procesos: calidad, gestión humana y logística.

\subsection{Iniciar}

\subsubsection{Sensibilizar}

La sensibilización tuvo como propósito justificar el cambio en la organización, dar a conocer los objetivos del evento kaizen, motivar la participación de trabajadores, evidenciar las posibilidades de aprendizaje y la alineación de esta estrategia con el sistema de gestión de la compañía. Este momento metodológico consumió un mes, llevando a cabo cuatro sesiones, presenciales, de dos horas cada una. En ellas se impartieron los siguientes temas: el sistema de gestión de la empresa, el lado humano (alinear objetivos individuales con los de la empresa, conciliar intereses de roles diferentes, integrar al equipo hacia la visión de la empresa, barreras por enfrentar, virus de la actitud, y lenguaje en común).

\subsubsection{Configurar el equipo}

Luego, con los trabajadores interesados en participar, se formaron dos equipos. El primer equipo se denominó "Proyección innovadora", y estuvo integrado por tres ensambladores de la línea A y uno de la B, y un líder operativo de la línea B. El segundo equipo tuvo por nombre "Reformas continuas", y estuvo conformado por un auxiliar administrativo, una auxiliar de almacén, la recepcionista, un auxiliar de mantenimiento y un ensamblador de la línea $A$.

\subsubsection{Capacitar al equipo}

La capacitación se llevo a cabo a través de un híbrido entre sesiones magistrales y estrategias de aprendizaje activo (colaborativo y basado en problemas). Se usaron lúdicas como "El avión de la muda" (PÉREZ, 2011a), que consiste en simular una empresa de producción de aviones (papel) en la que los participantes (personal de la empresa) ejercen roles específicos en la fabricación, incluyendo ensambladores e inspector de línea. A estos les correspondió fabricar 10 aviones en un tiempo límite de 2,5 minutos bajo un escenario inicial caracterizado por abundantes mudas. Al final se identifican los desperdicios de manufactura, se generan e implementan ideas de mejoramiento, la mayoría relacionadas con las 5S, logrando así la meta de producción. Para mayor información consultar "El avión de la muda” en (PÉREZ, 2011a).

También se desplegaron sesiones de capacitación en estadística, empleando naipes, dominó, entre otros juegos; a través de ellos se entrenó en: diagrama de Pareto, hoja de verificación, estratificación, espina de pescado, cinco por qué, histograma, gráfico de tendencia. Duración: 24 horas: 50\% presencial, 50\% trabajo extra.

Puede verse, en este momento metodológico, la incorporación de la actividad lúdica, la cual permite vincular emociones, toma de decisiones, habilidades comunicativas y pensamiento creativo en un entorno simulado (PÉREZ; JARAMILLO; MORENO, 2010; PÉREZ, 2012). De esta manera se convierte en un importante mecanismo para generar mayores niveles de confianza y cooperación en el equipo, lo cual contribuye a potenciar la construcción de conocimiento significativo, según lo exponen teorías sobre aprendizaje colaborativo y basado en problemas.

\subsection{Desarrollar}

\subsubsection{Muda}

Mediante lluvia de ideas se definieron y priorizaron los muda a intervenir; uno para cada equipo, así: en la línea A se genera frecuentemente muda de 


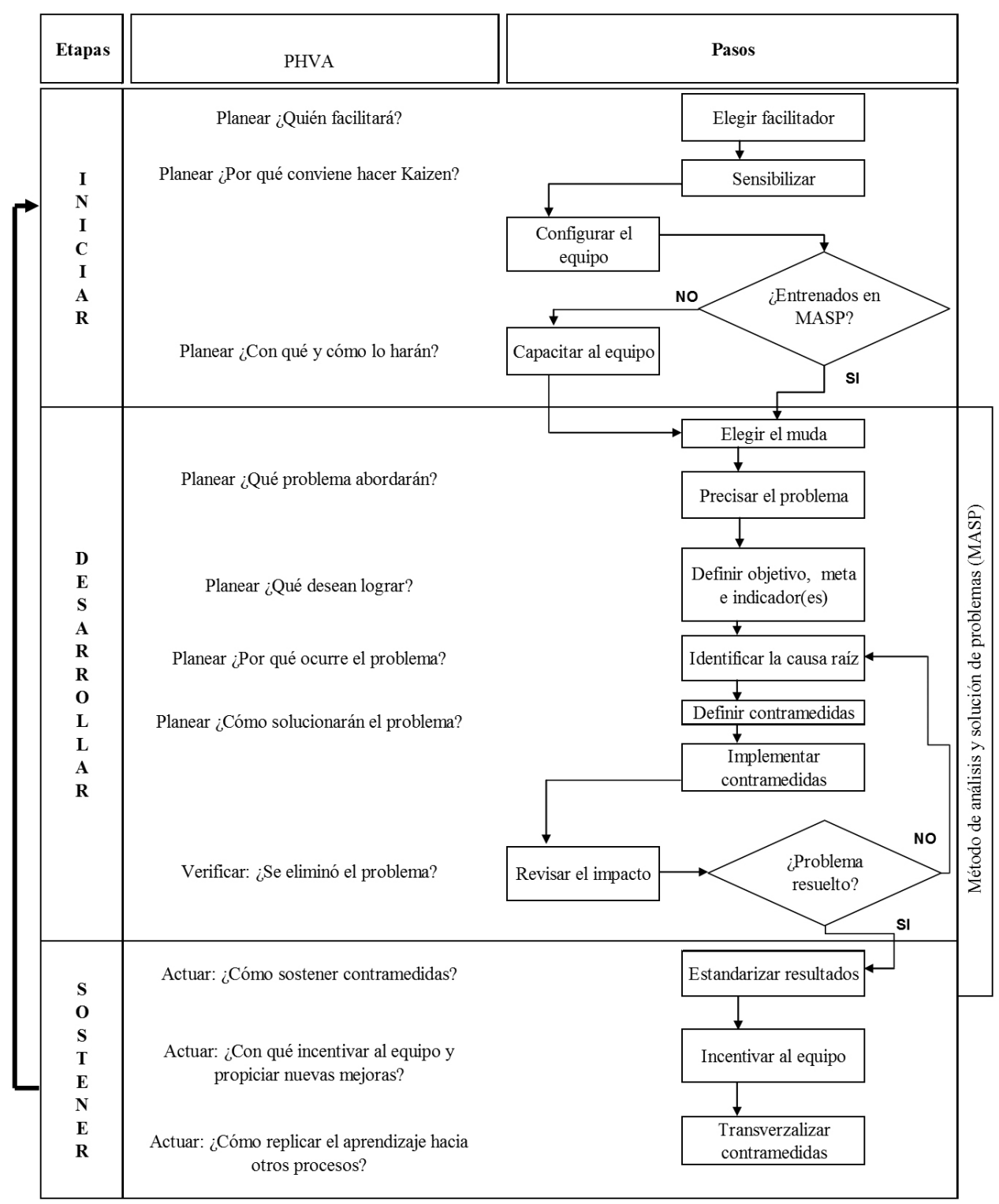

Figura 1. Metodología propuesta para la implementación de eventos kaizen.

espera por falta de suministro (caso 1, Proyección innovadora) y defecto de hojas que se imprimen desde los procesos administrativos de la empresa (caso 2, Reformas continuas).

\subsubsection{Problema preciso}

Luego se procedió a precisar el problema para cada caso, pretendiendo responder a las incógnitas: qué, cómo, dónde, cuándo, y cuánto. Para ello, los mismos trabajadores elaboraron hojas de verificación y recolectaron datos durante un mes. (Ver evidencia de una de las hojas originales en la Figura 2).
Luego de tabular los datos y de aplicar lo aprendido en la etapa "Iniciar" (gráficas de Pareto, promedios, costos, etc), y de analizar los resultados con apoyo del equipo investigador, fue posible precisar los casos objeto de estudio, así: Caso 1: "aproximadamente cada dos días la línea A para por falta de material SSP (82\% de ocurrencia frente a otros motivos de paro); en el 78\% de los casos ocurre durante la producción de la referencia 3P (entre las 10am y las $12 \mathrm{md}$ ). En promedio, dicho muda de espera tarda una hora y 21 minutos, generando un costo anual de tiempo improductivo de \$5'772.416". SSP es un término que 
representa piezas que exporta directamente la empresa cliente (ensambladora), las almacena y luego las envía a sus proveedores autopartistas para la integración.

Caso 2: "en la empresa $A B C$, se presenta muda de defectos de hojas impresas (promedio 38 diarias), generando un costo anual de \$1'368.000, donde el asistente de despachos, el responsable de calidad y el Jefe de mejoramiento, son los que más inciden en dicho desperdicio (media de 30 hojas día), arrojando estos tres colaboradores alrededor del 79\% del citado costo (\$1’080.000)”.

\subsubsection{Objetivos, metas e indicadores}

Teniendo presente la situación inicial, los colaboradores definieron los objetivos, metas e indicadores de seguimiento para ambos casos: (1) "reducir mínimo en 50\% el muda de espera de SSP,

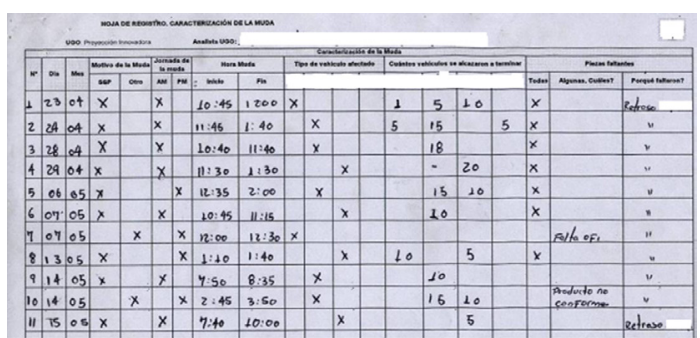

Figura 2. Extracto de una de las hojas de verificación diligenciadas manualmente por los colaboradores operativos, integrantes del equipo "proyección innovadora" (se agregó color blanco en información confidencial). que afronta la línea A". Indicadores: ocurrencia media diaria del muda por SSP, duración media y costo anual. (2) "disminuir, en promedio a 22, la cantidad diaria de hojas impresas con muda de defectos, reduciendo así el costo anual de dicho muda de defectos. Indicadores: promedio diario de hojas defectuosas y costo anual".

\subsubsection{Causas}

Este momento metodológico partió de una lluvia de ideas para explorar posibles causas de los diferentes muda; esto dio lugar a diagramas espina de pescado. Luego de seleccionar las ideas más probables, se profundizó con la técnica de los cinco por qué. A modo de evidencia, y por criterios de extensión de manuscrito, en la Figura 3 se presenta el diagrama de espina de pescado para el caso 2.

Para el caso 1, cuya causa antes era atribuida a "la culpa es del cliente, que teniendo el SSP, no lo envía a tiempo, por eso toca esperar", se encontró como causa raíz "la línea A tiene más personal del que necesita, produciendo más rápido de lo que requiere el cliente y agotando entre las 10:00 AM y las 12:00 PM (producción del vehículo 3P), el stock de SSP antes de tiempo"; en otras palabras, no se había considerado necesario alinear la producción con la demanda, lo cual generaba el muda de espera por falta de SSP.

Para el caso 2 "La impresora vino mala" el análisis realizado llevó a identificar tres causas raíz: 1. "No se ha considerado necesario informar al colaborador qué tamaño de hoja configurar, cómo y en qué momento

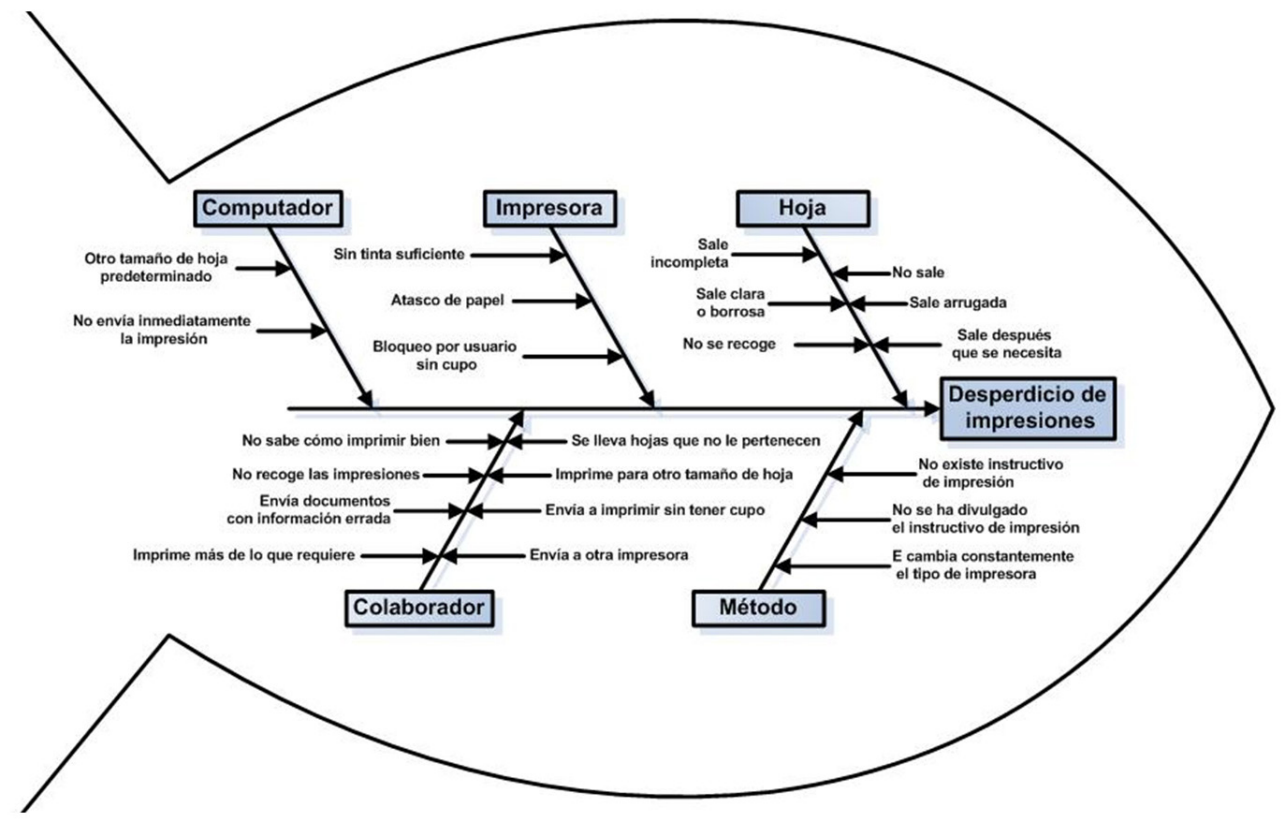

Figura 3. Espina de pescado para causas probables del caso 2. 
hacerlo"; ello ocasionaba que las hojas se imprimieran incompletas (margen, palabras, etc). 2. "No se había considerado necesario definir un lugar y momento para archivar las hojas impresas que aún no han sido recolectadas por los usuarios. Consecuencia: cuando el colaborador iba a recoger las hojas no las encontraba y volvía a enviarlas a imprimir, ya que el primero que las encontraba en despachos, las almacenaba donde hubiese algún espacio. 3. "fuertes vientos penetraban hacia la impresora y movían de lugar las hojas antes de que el usuario las recogiera. Consecuencia: hojas en el piso sucias o desaparecidas.

\subsubsection{Contramedidas}

Luego, se definieron las acciones de mejora. Para el caso 1 fue buscar una mejor alineación entre la producción de la cojinería para el vehículo 3P con la demanda del cliente. Dicha alineación fue fruto de una propuesta consensuada, basada en sentido común y en la experiencia y opinión de los mismos trabajadores operativos del equipo, consultándola luego con la jefa de producción y con el supervisor de producción de la empresa ABC. La Figura 4 muestra
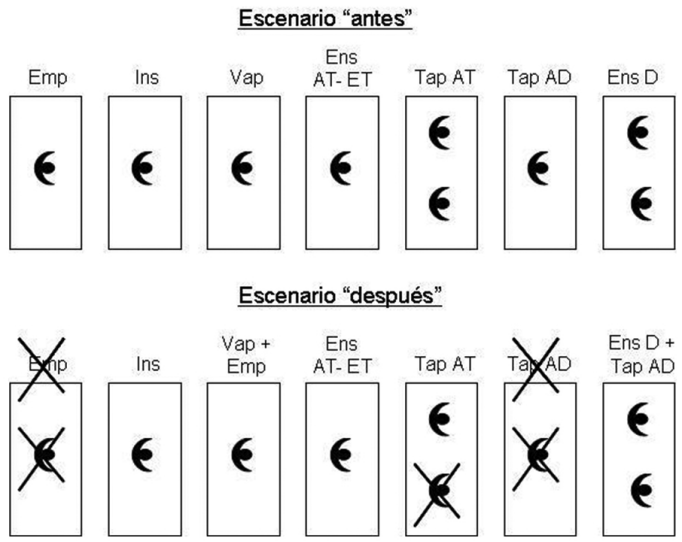

Figura 4. Línea de producción A, antes y después de la contramedida. la estructura de la línea "A" en los escenarios antes y después de implementar la contramedida.

Para el caso 2 se definieron las siguientes acciones, fruto de lluvia de ideas entre los trabajadores: 1 . Elaborar, divulgar y capacitar al jefe de mejoramiento, responsable de calidad y al despachos en un instructivo de configuración de hoja en el computador para impresión. 2. Diseñar, divulgar y capacitar en un procedimiento que defina el responsable, el lugar y el momento en que deben archivarse las hojas impresas, que aún no han sido recolectadas por los usuarios. 3. Diseño y fabricación de una cubierta en MDF para evitar que el viento se lleve las hojas impresas.

La Figura 5 da evidencia de los escenarios antes y después de haber implementado las contramedidas para la impresora.

\subsubsection{Impacto}

Los resultados para ambos casos fueron monitoreados durante un mes (julio de 2008). En la Tabla 1 se exponen las características cuantitativas que evidencian el impacto de la contramedida en el caso 1.

Para el caso 2 el impacto directo en la cantidad de hojas impresas defectuosas se muestra en la Tabla 2.

\subsubsection{Estandarización de resultados}

Tras verificar el impacto y observar la disminución del problema se procedió a estandarizar; para el primer caso se hizo actualización de las gamas de proceso de la línea $\mathrm{A}$, bajo los elementos de un $5 \mathrm{~W}+2 \mathrm{H}$ : ¿Qué?, ¿Por qué?, ¿Quién?, ¿Cómo?, ¿Cuándo?, ¿Dónde?, ¿Con qué?, cuya implementación dentro del sistema de gestión de la calidad (SGC) de la compañía estuvo a cargo de la Jefe de producción. Para el segundo caso se procedió así: tareas de actualización del SGC con el instructivo de configuración y el de recolección y ubicación de hojas impresas, empleando: ¿Qué?, ¿Quién?, ¿Cómo?, ¿Cuándo?, ¿Dónde?, acompañando este último con gerencia visual en la zona de despachos.

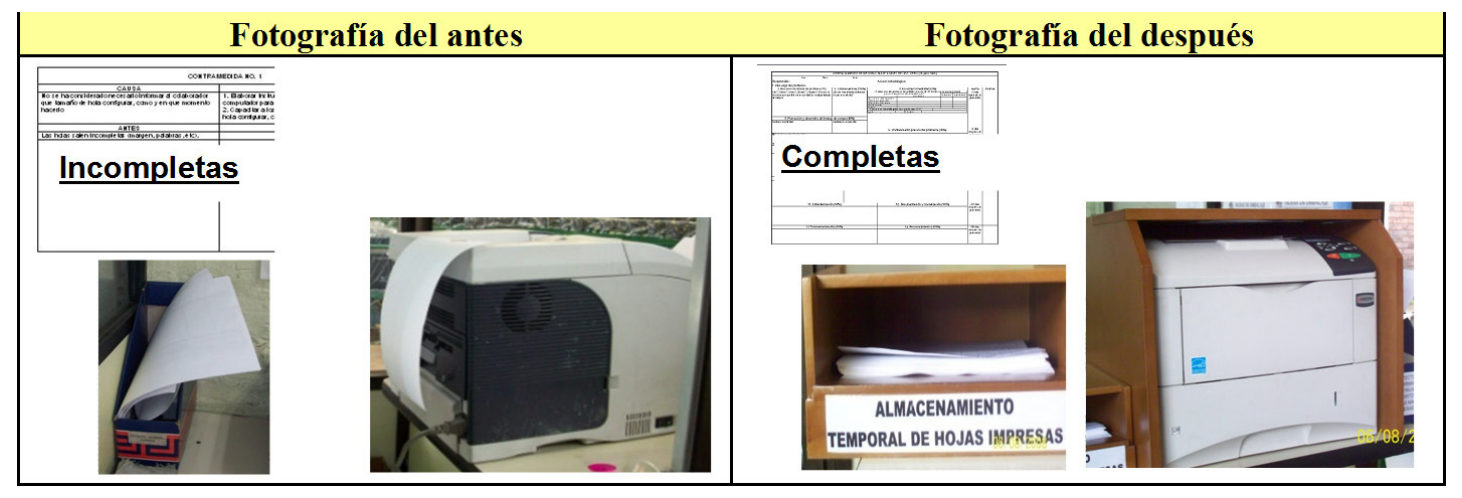

Figura 5. Impresora antes y después de las contramedidas. 
Tabla 1. Resultados antes y después de implementar la contramedida.

\begin{tabular}{lccc}
\multicolumn{1}{c}{ Características } & Antes & Después & Variación porcentual (\%) \\
\hline Número de centros de trabajo & 7 & 5 & $-28,57$ \\
Número de trabajadores & 9 & 6 & $-33,33$ \\
Ocurrencia promedio diaria del muda & 1,6 días & 0,08 días & -95 \\
Duración promedio del muda & 52 minutos & 6,75 minutos & -87 \\
Producción de sillas por cada trabajador & 3,89 & 4,17 & 7,19 \\
Costo anual del muda de espera & $5,772.319$ & 749.291 & -87 \\
\hline
\end{tabular}

Tabla 2. Resultados antes y después de implementar las contramedidas.

\begin{tabular}{lccc}
\multicolumn{1}{c}{ Caracteristicas } & Antes & Después & Variación porcentual (\%) \\
\hline Promedio diario de hojas impresas defectuosas. & 38 & 15 & $-60,52$ \\
Costo anual del muda de desperdicio & 1 '368.000 & 540.000 & $-60,52$ \\
\hline
\end{tabular}

\subsubsection{Incentivo al equipo}

Para ambos casos se brindo felicitación pública, diploma de graduación en la metodología kaizen para cada miembro de los equipos, participación en la Primera Convención Kaizen de la empresa ABC y bono para compra en almacén de cadena.

\subsubsection{Transverzalización de contramedidas}

Para el caso 1 se realizó un estudio formal de balanceo para la línea, que puede consultarse en (PÉREZ; PARRA, 2010). Y para el caso 2 se acordó como tarea capacitar a todas las personas involucradas con la impresión de hojas, ya que el proyecto solo abordó a los tres que más generaban defectos: jefe de mejoramiento, responsable de calidad y despachos.

\section{Discusión}

Este trabajo partió de la búsqueda de soluciones a dos problemas relacionados con desperdicios de manufactura, uno de ellos de espera y el otro de defectos. En un primer acercamiento a las causas de ambos muda, con base en las opiniones de personal de la empresa, se encontró que a priori, sin fundamento en análisis objetivo de causas, se tendía a dirigir la responsabilidad a factores externos, mostrando la posible solución del fenómeno de estudio como un trabajo que amerita intervención en el cliente externo (para el caso de muda de espera de SSP) y en el proveedor de impresoras (muda de defectos de hojas impresas). Ambas situaciones reflejaban imposibilidad de control interno y son coherentes con los postulados teóricos propuestos por Senge (2005), cuando se refiere a la tendencia de algunas organizaciones, de trasladar las causas de sus problemas a un "enemigo externo"; no obstante, para que fuese válido cualificar los dos citados fenómenos como el síndrome del "enemigo externo" en la empresa $A B C$, era preciso demostrar, con base en datos y hechos, que las causas raíz de dichos muda, en lugar de atribuirse a factores externos, se deben a causas viables de intervenir al interior de la organización.

De este modo, dando respuesta a la primera pregunta de investigación, la cual se orienta a determinar las causas raíz de los muda objeto de estudio, luego de desplegar las etapas iniciar y desarrollar, pudo comprobarse que la causa del muda de espera por falta de SSP fue el desbalance de la línea de producción, presentando más personal del que requiere. Para el caso del muda de defectos de hojas impresas, se encontró que la omisión de configuración de la impresora, ausencia de un lugar para alojar las hojas y los vientos que se llevan las hojas impresas, fueron los principales causantes del problema. Estos resultados, además, permitieron dar respuesta a la segunda pregunta de investigación ¿éstas causas se deben a factores externos o internos a la empresa?, teniendo evidencia para concluir que las causas son asignables a factores internos, posibles de intervenir con el talento humano y con los recursos de la empresa $A B C$.

La búsqueda de estos resultados comenzó con la manifestación de garantías de apoyo desde la dirección de la empresa y con el despliegue de los tres pasos de la etapa iniciar, orientados a la preparación del evento kaizen (metas, participantes y pre-diagnóstico) (SHERIDAN, 1997; BATEMAN, 2005). Lo anterior, fue debido a las recomendaciones de Landa (2009) para los factores de éxito de eventos kaizen, de Farris et al. (2008) sobre características de los fracasos de los mismos, de Imai (1997) y Cuscela (1998) acerca de la importancia de entrenar el equipo de trabajo, de Japan Human Relations Association (1990), Landa (2009) y Senge (2005), respecto a la necesidad de ambientes que propicien la participación y compromiso de los empleados; todo ello, complementado con las experiencias exitosas desde el aprendizaje activo (PÉREZ, 2012).

Esta etapa, denominada iniciar, que en casos puede verse innecesaria para seguir metodológicamente las etapas del MASP, sobre todo en cuanto a la sesión de sensibilización, puede ser determinante en el éxito o fracaso de los eventos kaizen, a lo largo del 
tiempo, al considerar la evidencia arrojada por la citada literatura y la distancia entre causa-efecto en espacio y tiempo, que puede ser necesaria para conocer el verdadero impacto de dichos eventos, más aún, cuanto a pesar de que se intervengan problemas duros (objetivos precisos, método preestablecido, una misma mirada, ...) la necesidad de sostener compromiso, motivación, creatividad, aprendizaje y participación de los empleados, se constituye en un problema blando (varias interpretaciones dependiendo del observador,...).

La siguiente fase llevada a cabo para responder a las dos primeras preguntas de investigación fue desarrollar, hasta el paso de identificar causas. Allí, para precisar el problema objeto de estudio, que de acuerdo con los apartados teóricos de la metodología de la investigación, toda idea al inicio es vaga (HERNÁNDEZ; FERNÁNDEZ; BAPTISTA, 1997), se procedió a planear y realizar el trabajo de campo para que el personal de los equipos kaizen se introdujeran en el ámbito de conocimiento de donde provienen los muda. En ambos casos: espera por falta de suministro y defecto de hojas, los mismos empleados utilizaron hojas de verificación estratificadas, construyeron diagramas de Pareto y calcularon estadísticas básicas como el promedio. Ello permitió precisar cualitativamente el problema, pero además, cuantificarlo con base en información objetiva, como lo recomienda Imai (2001), siendo viable, a partir de dicha información, definir adónde se quiere llegar (objetivo) y su cuantificación (meta). Con el problema y la situación deseada precisos, los equipos kaizen iniciaron la construcción de diagramas Pareto para identificar causas potenciales, las cuales luego fueron estudiadas desde la herramienta de los cinco por qué, permitiendo llegar, luego de preguntarse ocho veces en el caso 1 y de 10 veces en el caso 2 , a las posibles causas raíz de los muda observados.

Respondiendo a la tercera pregunta de investigación ¿cómo intervenir dichas causas, a fin de eliminar o reducir la recurrencia de dichos muda?, se dio continuidad al macroproceso desarrollar, partiendo de la definición de contramedidas. En el caso 1, asociado al muda de espera por falta de SSP, la solución desde el punto de vista de la ingeniería sería balancear la línea de producción, para lo cual hubiese sido necesario estimar el tiempo de ciclo de cada centro de trabajo, de la línea completa y del takt time; no obstante, esta contramedida carecería de los principios que caracterizan una acción de mejoramiento "paso a paso", propuesta, liderada y desplegada por los mismos empleados del nivel operativo, la cual debe considerar: simplicidad, sentido común, creatividad, participación de los empleados y motivación (OHNO, 1987; IMAI, 2001; WOMACK, JONES; ROOS, 1990; ALUKAL, 2007; MANOS, 2007); por ello, la definición de las contramedidas, para ambos casos ( 1 y 2), surge desde los mismos equipos kaizen, producto de la exposición de sus ideas de mejora, las cuales se basan, entre otros, en sus modelos mentales, experiencia, hallazgos encontrados, recursos y aprendizaje adquirido (HUBER, 2008), llegando luego a un consenso al desplegar la priorización de propuestas a través de la herramienta de lluvia de ideas.

Otra evidencia de la simplicidad, creatividad y sentido común puede notarse en las contramedidas del caso 2, donde en una de ellas los mismos trabajadores, empleando material de la empresa, construyeron un cubículo para alojar la impresora y evitar que las hojas impresas sean retiradas por el viento al que estaban expuestas Figura 3). Vale anotar que, en ambos casos (1 y 2), para llegar al consenso, los mismos empleados solicitaron conocer la opinión de otras personas de la empresa, entre ellas, la Jefa de producción y la de Gestión humana.

Las contramedidas elegidas (Figuras 2 y 3 ) desde el punto de vista de la metodología de la investigación, pueden asociarse a hipótesis de trabajo, ya que los dos equipos kaizen tienen la creencia, apoyados entre otros, en información objetiva, de que al implementarlas en la empresa resolverían los problemas estudiados, pero para ello, era preciso, luego de implementar dichas iniciativas, dar un tiempo de monitoreo del impacto arrojado por las mismas. En ambos casos de estudio ( 1 y 2), atendiendo a restricciones de tiempo por parte de la empresa, el periodo de observación abordó el mes de julio de 2008. El método seguido en el caso 1 (muda de espera por falta de SSP) fue el monitoreo del tablero de indicadores de la planta, donde se registra, entre otros, los paros de la línea A y el motivo de los mismos; para el caso 2 (defecto de hojas impresas) fue necesario implementar la misma estrategia de recolección de datos llevada a cabo para precisar el problema, la cual consistió en el registro, al final del turno (4:00 PM), de la cantidad de hojas impresas defectuosas. Luego del registro, la cuantificación de los resultados obtenidos reflejó progreso en cuanto a la búsqueda de soluciones a los problemas estudiados, en el sentido de que en el caso 1 , la ocurrencia diaria y la duración, atribuidas al muda de espera por falta de SSP, se vieron disminuidas, estimando una reducción del 87\% en el costo anual generado por el tiempo improductivo (Tabla 2). Además de ello, la contramedida impactó otro indicador no considerado en la planeación inicial y fue debido a que la integración de operaciones realizada a la línea A, llevó a eliminar dos centros de trabajo y a reducir tres trabajadores durante la producción de la cojinería del vehículo 3P; esto incrementó la capacidad de producción (vehículos/hombre) en 7\% (Tabla 2). Según información de la empresa, algunos de estos trabajadores pasarían a otras líneas; respecto a ello, es de resaltar que, considerando los stakeholders de la organización de hoy: clientes, empleados, accionistas, proveedores y sociedad (ANDRIANI; BIASCA; RODRÍGUEZ, 2003), donde la empresa debe 
velar por satisfacer las necesidades del conjunto, este último impacto de la contramedida podría generar beneficio para unas caras del sistema y perjudicar a otras, de ahí la importancia de reflexionar sobre la “solución óptima sistémica” (PÉREZ, 2011b). Estas decisiones se salen del control del equipo investigador.

Dado que el impacto de las contramedidas fue considerado por la empresa como satisfactorio, la finalización del MASP se hizo con la estandarización de las mismas, incluyendo la exhibición de los resultados, según lo presentan autores como Agudelo y Escobar (2007). Estos son los últimos pasos del MASP, resaltando que autores como Bateman y David (2002), haciendo uso del seguimiento de acciones inconclusas, generalmente por tres meses, dan por culminado el evento kaizen.

Es de destacar que durante la etapa desarrollar, los participantes afrontaron un aprendizaje soportado en teorías constructivistas, específicamente mediante los aprendizajes colaborativo y basado en problemas. En ese sentido, se presentó a los trabajadores un caso problema, que fue tomado como un reto para los mismos, y que a su vez, les despertó interés; para solucionarlo realizaron: análisis del problema, generaron hipótesis, identificaron vacíos de conocimiento, fijaron metas (HUBER, 2008), intercambiaron opiniones, compartieron conocimiento, soportaron críticas y adoptaron un pensamiento crítico, llevándolos a reflexionar sobre ¿qué problemas encontraron?, ¿cuáles son sus causas? y ¿cómo eliminarlas?, acordes con el ciclo de la exploración (BRANDA, 2001).

Como puede verse en la Figura 1, a pesar de que, en la metodología propuesta, la estandarización corresponde al último paso del MASP, que inicia en la etapa desarrollar, ésta se circunscribe en el inicio de la etapa sostener, tendiente a posibilitar que las contramedidas se mantengan en el tiempo para prevenir la recurrencia de los problemas. Además de lo anterior, la metodología empleada considera la necesidad de garantizar el sostenimiento de la motivación de los trabajadores que participaron en los eventos kaizen y de animar hacia la participación en eventos futuros, no solo por parte de los que actualmente intervinieron, sino de los demás empleados. Esto se fundamenta en estudios como Cheser (1998), donde se encuentra la correlación positiva entre la motivación y la aplicación del kaizen y, en Japan Human Relations Association (1990), que resalta los mecanismos de recompensas (preferiblemente no monetario) para potenciar la interiorización y práctica de los eventos kaizen. Para ambos casos ( 1 y 2), la empresa ABC, como método de recompensa, empleó: felicitación pública, diploma de graduación en la metodología de trabajo, participación en la Primera Convención Kaizen e incentivo monetario.

Finalmente, para facilitar el proceso de lecciones aprendidas, fruto del aprendizaje organizacional que dejaron los dos casos, sigue un paso denominado transversalizar, el cual considera el estudio por parte de personal táctico o estratégico de la empresa, de posibles líneas, proceso u objetos de estudio, diferentes a los que se intervino, que puedan ser vulnerables ante las causas de los problemas abordados, a fin de implementarles también las contramedidas. En ese sentido, con relación al caso 1 , la empresa detectó posibilidad de desbalance en otras líneas de producción, abriendo espacio para la realización de un nuevo proyecto, el cual fue intervenido bajo un enfoque más especializado, fuera de la modalidad de eventos kaizen, empleando elementos de la simulación computacional (PÉREZ; PARRA, 2010).

\section{Conclusiones}

En la búsqueda de soluciones a dos problemas de una firma autopartista, relacionados con muda de espera por falta de suministro y de defectos de hojas impresas, se hace una propuesta metodológica para adoptar y desplegar la filosofía de los eventos kaizen. Esta propuesta está conformada por tres etapas (iniciar, desarrollar y sostener), las cuales forman un ciclo que motiva a finalizar un determinado evento y preparar el entorno para el siguiente. Se apoya en elementos teóricos del kaizen, desde el enfoque japonés, como filosofía, y desde el enfoque occidental, como técnica de solución de problemas.

La metodología seguida, antes de desplegar propiamente el análisis y la solución del problema, destaca la necesidad de una preparación de las personas en la fase de iniciar, en favor de mayores posibilidades de lograr el compromiso y la participación activa de los empleados, luego viene la fase desarrollar, donde se ejecuta propiamente el MASP bajo un enfoque de aprendizaje colaborativo y basado en problemas; finalmente, viene la fase sostener, en la cual se busca mantener las contramedidas a lo largo del tiempo, evitar la recurrencia del problema e incentivar a los participantes a través de mecanismos de recompensas, de modo que se potencie de nuevo el inicio de la metodología. Durante todo el despliegue del trabajo, se resalta la necesidad del compromiso de la alta dirección y de condiciones de la cultura organizacional, sugeridas por la literatura, propicias para incrementar la posibilidad de éxito de los eventos kaizen.

A nivel pragmático, el despliegue de la metodología permitió identificar las causas de los muda objeto de estudio (tiempo de espera y defectos). Estos se debieron a factores internos a la empresa, contrario a las creencias iniciales de la firma sobre causas externas, no controlables, que hacían alusión al síndrome del "enemigo externo". Al reunir evidencia sobre las posibilidades de control desde la misma firma, en los dos casos específicos pudo definirse e implementarse contramedidas basadas en creatividad, 
simplicidad, sentido común, consenso y participación de los empleados, para eliminar o reducir dichos muda.

El impacto arrojado con el despliegue de la propuesta metodológica se dedujo satisfactorio respecto al mejoramiento de indicadores asociados a los problemas precisos, entre ellos, costos anuales de los muda de interés; no obstante, en uno de ellos se identificó la posibilidad de cumplirle al cliente con tres trabajadores menos, lo cual hace viable que al final, no todas las "caras" del sistema se hayan sentido satisfechas, de ahí la necesidad de que los efectos de las soluciones de un problema específico mediante esta propuesta metodológica sean complementados con una visión sistémica, procurando no afectar otras partes interesadas. Este trabajo es útil para docentes, estudiantes, empresarios y consultores, en la medida en que ofrece elementos metodológicos, que brinda la posibilidad de adaptación a diversos contextos empresariales, exigiendo aspectos de la gestión organizacional (compromiso directivo, justificación del cambio, entrenamiento de empleados, incentivos, comunicación, estrategias de aprendizaje, entre otros) para posibilitar la solución de los problemas que se aborden y el sostenimiento de la práctica de los eventos kaizen.

Como trabajo futuro surge la necesidad de evaluar, con rigor cuantitativo, el impacto arrojado por la etapa iniciar; para ello, es preciso el desarrollo y/o la adaptación de instrumentos que permitan medir cuantitativamente, qué tanto dicho proceso, en su configuración actual, impacta el trasfondo emocional de los empleados, propiciando la concientización, la motivación y las habilidades para desplegar la etapa desarrollar. Así mismo, la evaluación de la etapa sostener, considerando la interiorización de los resultados de los eventos kaizen, mediante la observación de las contramedidas durante mayores periodos de tiempo.

\section{Agradecimientos}

Los autores agradecen a la firma autopartista que propició el despliegue, tipo estudio de caso, de la metodología aquí propuesta. Se resalta el compromiso de dicha empresa con la mejora continua, en favor de: clientes, empleados, accionistas, proveedores y sociedad en general. De esta misma firma, especial agradecimiento a la Gerencia general, al equipo de colaboradores operativos que participaron en el despliegue, a la Dirección de planta, a la Jefa de producción y a la de Gestión Humana. Agradecimientos a los evaluadores del artículo y a la Asociación Brasileña de Ingeniería de Producción (ABEPRO).

\section{Referencias}

AGUDELO, L.; ESCOBAR, J. Gestión por procesos. 2. ed. Editorial los autores, 2007.
AL-TAHAT, M.; ETEIR, M. Investigation of the potential of implementing Kaizen principles in Jordanian companies. International Journal of Product Development, v. 10 n. 1-3, p. 87-100, 2010. http://dx.doi.org/10.1504/ IJPD.2010.029987

ALUKAL, G. Lean Kaizen in the 21st century. Quality Progress, v. 40, n. 8, p. 69-70, 2007.

ANDRIANI, C.; BIASCA, R.; RODRÍGUEZ, M. Un nuevo sistema de gestión para lograr PYMES de clase mundial. Ed. Norma, 2003.

AOKl, K. Transferring kaizen activities to overseas plants in China. International Journal of Operations and production Management, v. 28, n. 6, p. 518-539, 2008. http://dx.doi.org/10.1108/01443570810875340

BARRAZA, M.; SMITH, T.; DAHLGARD, S. The impact of Kaizen Events on improving the performance of automotive components first-tier suppliers. International Journal of Automotive Technology and Management, v. 9, n. 4, p. 362-376, 2009.

BARRAZA, M.; SMITH, T.; DAHLGARD, S. Lean-Kaizen public service: An empirical approach in Spanish local governments. TQM Journal, v. 21, n. 2, p. 143-167, 2009. http://dx.doi.org/10.1108/17542730910938146

BATEMAN, N.; DAVID, A. Process improvement programmes: a model for assessing sustainability. International Journal of Operations \& Production Management, v. 22, n. 5, p. 515-526, 2002. http://dx.doi. org/10.1108/01443570210425156

BATEMAN, N. Sustainability: the elusive element of process improvement. International Journal of Operations \& Production Management, v. 25, n. 3, p. 261-276, 2005. http://dx.doi.org/10.1108/01443570510581862

BESSANT, J. High-Involvement Innovation. Chichester: John Wiley and Songs Ltd, 2003.

BRANDA, L. Aprendizaje basado en problemas, centrado en el estudiante, orientado a la comunidad. In: JORNADAS DE CAMBIO CURRICULAR DE LA FACULTAD DE MEDICINA DE LA UNIVERSIDAD DE BUENOS AIRES, 2001, Buenos Aires. Anales... Buenos Aires: Organi\#zación Panamericana de la Salud, 2001. p. 79-101.

BRUNET, P.; NEW, S. Kaizen in Japan: an empirical study. International Journal of Operations \& Production Management, v. 23, n. 12, p. 1426-1446, 2003. http:// dx.doi.org/10.1108/01443570310506704

CHESER, R. The effect of Japanese Kaizen on employee motivation in US manufacturing. International Journal of Organizational Analysis, v. 6, n. 3, p. 197-217, 1998. http://dx.doi.org/10.1108/eb028884

CODINA, L. Scopus: el mayor navegador científico de la Web. El profesional de la información, v. 14, n. 1, p. 4449, 2005. http://dx.doi.org/10.3145/epi.2005.feb.07

CUSCELA, K. Kaizen blitz attacks work processes at Dana Corp. llE. Solutions, v. 30, n. 4, p. 29-31, 1998.

DOOLEN, T. et al. Kaizen events and organizational performance: A field study. International Journal of Productivity and Performance Management, v. 57, n. 8, p. 637-658, 2008. http://dx.doi. org/10.1108/17410400810916062

FARRIS, J. et al. Critical success factors for human resource outcomes in Kaizen events: An empirical study. International Journal of Production Economics, v. 117, n. 1, p. 42-65, 2009. http://dx.doi.org/10.1016/j. ijpe.2008.08.051

FARRIS, J. et al. Learning from less successful Kaizen events: A case study. EMJ - Engineering Management Journal, v. 20 , n. 3, p. 10-20, 2008. 
HERNÁNDEZ, R.; FERNÁNDEZ, C.; BAPTISTA, P. Metodología de la Investigación. México: McGraw-Hill, 1997.

HUBER, G. Active learning and methods of teaching. Revista de Educación, número extraordinario. Tiempos de cambio universitario en Europa, p. 59-81, 2008.

IMAl, M. Kaizen: the key to Japan's competitive success. New York: Random House, 1986.

IMAl, M. Gemba Kaizen: a commonsense, low-cost approach to management. New York: McGraw Hill, 1997.

IMAl, M. Kaizen: la clave de la ventaja competitiva japonesa. 13. reimpr. México: Compañía Editorial Continental, 2001.

JAPAN HUMAN RELATIONS ASSOCIATION. Kaizen Teian. Cambridge: Productivity Press, 1990.

LANDA, J. Factores de éxito y permanencia en eventos Kaizen. Aplicación en la industria maquiladora del norte de México. Sinnco, 2009. p. 1-20.

MANOS, A. The benefits of kaizen and kaizen events. Quality Progress, v. 40, n. 2, p. 47, 2007.

MARIN, J.; GARCIA, J.; BONAVIA, T. The impact of Kaizen Events on improving the performance of automotive components'first-tier suppliers. International Journal of Automotive Technology and Management, v. 9, n. 4, p. 362-376, 2009.

MURATA, K.; KATAYAMA, H. A study on construction of a kaizen case-base and its utilization: a case of visual management in fabrication and assembly shopfloors. International Journal of Production Research, v. 48, n. 24, p. 7265-7287, 2010. http://dx.doi. org/10.1080/00207540903373823

OHNO, T. The Toyota Production System. Beyond large scale production. New York: Productivity press, 1978.

PÉREZ, J.; JARAMILLO, P. Y.; MORENO, L. El comercio de canicas: herramienta de apoyo para la enseñanza- aprendizaje práctico de la programación lineal. Revista Facultad de Ingeniería UCV, v. 25, n. 1, p. 43-52, 2010.

PÉREZ, J.; PARRA, C. Mejoramiento de una línea de Ensamble de Asientos Delanteros Autopartistas usando Simulación Dinámica de Sistemas. Revista Técnica de Ingeniería, v. 33, n. 1, p. 11-20, 2010.

PÉREZ, J. El avión de la muda: herramienta de apoyo a la enseñanza-aprendizaje práctico de la manufactura esbelta. Revista Facultad de Ingeniería Universidad de Antioquia, n. 58, p. 173-182, 2011a.

PÉREZ, J. Modelación lineal en ingeniería industrial: una mirada sistémica. Medellín: Editorial Universidad de Antioquia, 2011b.

PÉREZ, J. Mejoramiento paso a paso en la PYME manufacturera ¿Cómo iniciar al personal operativo? Medellín: Editorial Universidad de Antioquia, 2012.

SENGE, P. La quinta disciplina: el arte y la práctica de la organización abierta al aprendizaje. 2. ed. 4. reimpr. Buenos Aires: Ediciones Granica S.A., 2005. p. 30-31.

SHERIDAN, J. Kaizen Blitz. Industry Week, v. 246, n. 16, p. 19-27, 1997.

SUÁREZ-BARRAZA, M. F.; MIGUEL-DÁVILA, J. Á. Encontrando al Kaizen: Un análisis teórico de la mejora continua. Revista Pecvnia, p. 285-311, 2008.

SUÁREZ-BARRAZA, M. F.; RAMIS-PUJOL, J. Implementation of Lean-Kaizen in the human resource service process: A case study in a Mexican public service organisation. Journal of Manufacturing Technology Management, v. 21, n. 3, p. 388-410, 2010. http://dx.doi. org/10.1108/17410381011024359

WITTENBERG, G. Kaizen - The many ways of getting better. Assembly Automation, v. 14, n. 4, p. 12- 17, 1994. http://dx.doi.org/10.1108/EUM0000000004213

WOMACK, J.; JONES, D.; ROOS, D. The Machine that changed the world. New York: Rawson Associates, 1990.

\title{
Overcoming the "external enemy" syndrome in an autoparts firm using kaizen
}

\begin{abstract}
Two problems in an autoparts firm are initially attributed to uncontrollable external factors ("external enemy" syndrome). This situation motivates the design of a methodology to guide kaizen event practices, supported by kaizen philosophy and methodology. The methodology consist of three steps: the first step, initiate, is designed to acquire employee commitment and participation and to develop initial employee skills. The second step, develop, analyzes the problem and implements the solution. The third process, sustain, is focused on sustaining the interventions, avoiding repetition of the problem and reinforcing the motivation of the participant. Deploying the methodology in both of the cases studied (1. Waiting time waste caused by a lack of supply and 2. Defects waste in printed sheets) allows to identify that the cause of those wastes were operational internal factors, instead of prior beliefs relating these facts to uncontrollable external reasons. The cause of waiting time was associated with the quantity of employees programmed into the assembly line, and the defective printings were related to storage and protection policies as well as the printing instructions. The impact of the interventions was satisfactory, because through the implementation of actions based on employee's creativity, common sense, consensus and participation, the problem's associated indicators are favored, including the waste's costs (Reduction of costs: $87 \%$ in waiting time costs; $60.5 \%$ in defective sheet costs). This study provides theoretical and methodological elements that make it adaptable to other contexts. The method requires top management commitment, employee comprehension of the change, employee training sessions, motivation and rewards, communication and learning strategies, among other factors, to solve problems and sustain the use of kaizen events.
\end{abstract}

Keywords

Kaizen. Applies research. Continuous improvement. Manufacturing wastes. 